PART IV

FUTURE EXPLORATIONS OF THE SOLAR SYSTEM 


\title{
SPECTROSCOPIC OBSERVATION OF VENUS
}

\author{
A. MONFILS and J. C. GÉRARD \\ Astrophysical Institute, University of Liège, Belgium
}

\begin{abstract}
The interest of a spectroscopic investigation of the upper atmosphere of Venus in the ultraviolet is briefly shown. The conditions for the observations to be made are defined and the main properties of the necessary instrument are deduced and described. The proposed package is a quadruple miniature spectrometer covering the entire spectral range extending from $1200 \AA$ to $8000 \AA$ with a resolving power of 500 .
\end{abstract}

\section{Introduction}

Venus is the third brightest object in the sky, but not much is known about it, especially if we compare it with the two brightest objects and with Mars. It can be said, in particular, that the structure and mechanisms of Venus' atmosphere are very little known.

Spectroscopy is a powerful tool which has provided, in the past, most of the information we now possess concerning celestial bodies. The spectra of Venus may permit :

- the identification, evaluation and localization of the concentration of minor components of the atmosphere

- the measurement of scale heights and their variations with solar illumination, cycles and activity

- the determination of the temperature of the upper layers; this requires a higher spectral resolution, but limited spectral ranges

- the detection, identification of any airglow and its correlation with solar activity. A comparison with the spectra obtained by Barth et al. (1971) in the case of Mars and his colleagues shows that at least one may expect to record:

- the Fox-Duffendack-Barker bands of $\mathrm{CO}_{2}^{+}$

- the $2900 \AA$ system of $\mathrm{CO}_{2}^{+}$

- the Cameron bands of $\mathrm{CO}$

- the comet-tail bands of $\mathrm{CO}^{+}$

$-\mathrm{L} \alpha$

- O I lines at 1305,1356 and $2972 \AA$

- C I line at $1657 \AA$

One can furthermore expect to detect other systems and lines such as the 5577 and $6300 \AA\left[\mathrm{OI}_{1}\right]$ lines and the $\mathrm{N}_{2}$ 1st and 2nd positive systems (or even rare gas emissions). On the other hand, $\mathrm{O}_{3}(2000-3000 \AA), \mathrm{CO}_{2}(1250-2000 \AA)$ and $\mathrm{CO}_{2}^{+}(3000-3500 \AA)$ may be observed in absorption.

\section{Conditions of Observation}

Numerous conditions of observation are possible. We have selected here what would appear to be the most simple and economic case: a satellite with an elliptical orbit perpendicular to the ecliptic plane, $24 \mathrm{~h}$ period, 300 or $1000 \mathrm{~km}$ perapsis. 


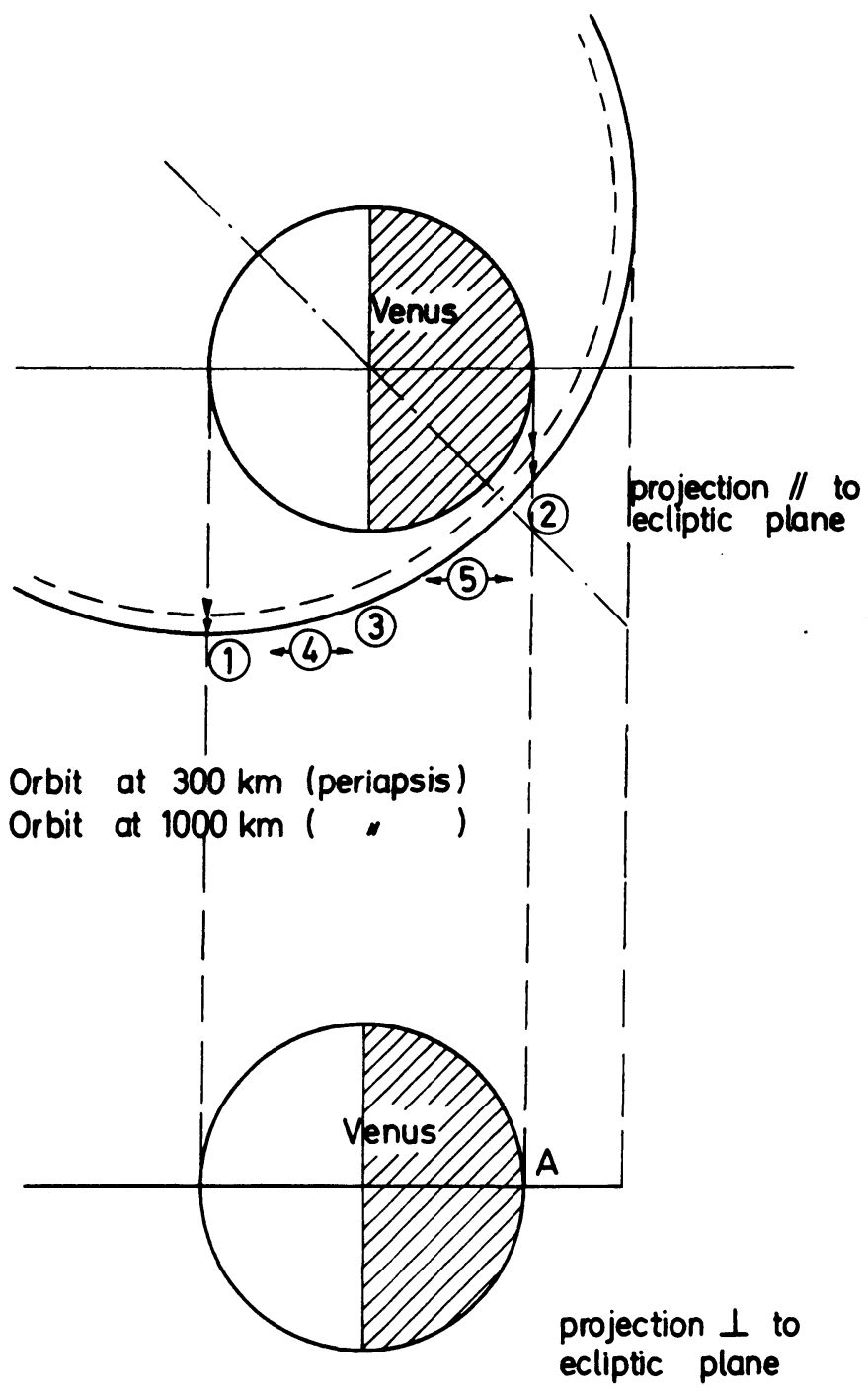

Fig. 1. Observational configurations in the case of an eccentric Venus Orbiter probe whose orbital plane is perpendicular to the ecliptic.

The observational configurations may be seen on Figure 1. In particular, the detection of some nightglow would be valuable in the perspective of the controversial ashenlight problem.

Four different satellite positions are of interest. The corresponding distances can be seen in Table I.

It can easily be shown that the observing times are of the order of 30 to $100 \mathrm{~s}$ for one profile. 
TABLE I

Conditions of observation

\begin{tabular}{|c|c|c|c|c|c|c|}
\hline \multirow[t]{2}{*}{ Inclination } & \multirow[t]{2}{*}{ Periapsis } & \multicolumn{5}{|c|}{ Approx. distances $(\mathrm{km})$} \\
\hline & & (1) & (2) & (3) & (4) & (5) \\
\hline $90^{\circ}$ & $\begin{array}{r}300 \mathrm{~km} \\
1000 \mathrm{~km}\end{array}$ & $\begin{array}{l}8000 \\
9000\end{array}$ & $\begin{array}{l}2600 \\
4000\end{array}$ & $\begin{array}{l}1400 \\
2200\end{array}$ & $\begin{array}{l}4500 \\
5200\end{array}$ & $\begin{array}{r}700 \\
1700\end{array}$ \\
\hline
\end{tabular}

\section{Spectroscopic Device}

A spectroscopic device designed for operation aboard a spinning probe orbiting around Venus should be compatible with:

- the very low weight available

- the fact that the image of the target is very probably spinning around the line of sight (in the best case).

- the very short observation periods (minutes of time)

- the low power and low transmission rate available

- the advantage of being able to modify the resolution in orbit.

Advantage should also be taken of the long overall lifetime of the mission.

\section{Angular Resolution of the Telescope}

This depends on the scale heights which may be readily evaluated. Taking the observing distances into account, it may be shown that an angular resolution of 1/200 rad is necessary. This settles the specifications of the telescope and simultaneously the diameter of the entrance hole of the spectrometer. The latter must be circular in order to maintain the spatial resolution independent of the attitude of the satellite. With a focal distance (F.D.) of $14 \mathrm{~cm}$ for the telescope, a circular hole of $0.7 \mathrm{~mm}$ diam maintains the desired spatial resolution.

\section{Optical Principles}

The luminosity of a spectrometer is proportional to the square of the F.D. (when the slit shape is constant). The weight is proportional to the cube of the F.D.

Consequently, the specific light gathering power per unit of weight is inversely proportional to the focal distance at constant weight, and is the main parameter. The total gathering power may then be recovered by multiplying the number of spectrographs; the resolving power may, in turn, be recovered by adding Fabry-Pérot type dispersing devices, and by using the gratings at the maximum number of lines per millimeter. This is made possible by the multiplication of the number of spectrometers, which helps here to keep each grating to a limited spectral range. As the latter are chosen so as to match the properties of the photocathodes, the whole system is optimized. 


\section{Description of the Experimental Package}

The package is typically composed of four parallel $9 \mathrm{~cm}$ F.D. Ebert-Fastie monochromators, each one following a $14 \mathrm{~cm}$ F.D. telescope (Figure 2). The four gratings are mounted on the same axis, and are simultaneously scanning their spectral range, which are given in Table II.

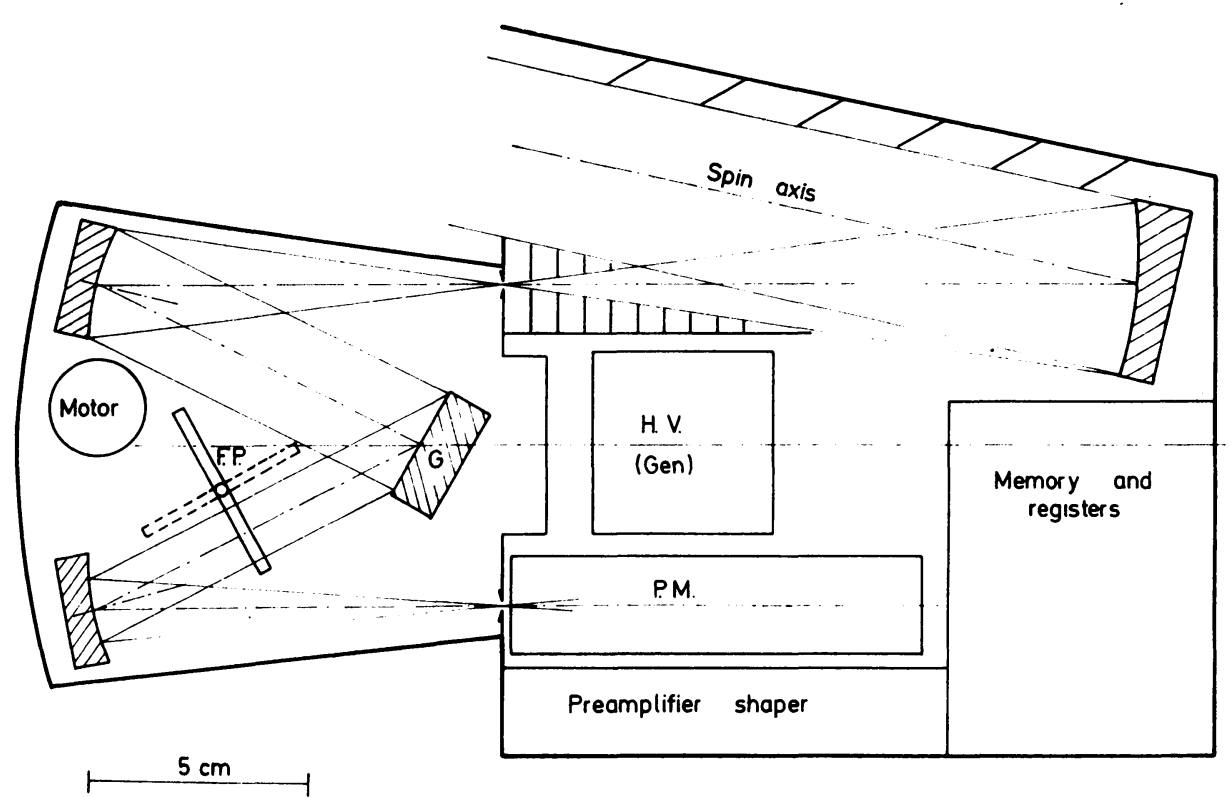

Fig. 2. View of the experimental package showing how the various optical and electronic constituents may be disposed. The package is typically composed of four such assemblies.

TABLE II

Gratings and their spectral range

\begin{tabular}{ll}
$\begin{array}{l}\text { Grating constants } \\
\left(\mathrm{mm}^{-1}\right)\end{array}$ & Wavelength ranges $(\AA)$ \\
\hline 6.000 & $1000-2000$ \\
3.600 & $1650-3300$ \\
2.400 & $2500-5000$ \\
1.500 & $4000-8000$
\end{tabular}

In the parallel beams, thin Fabry-Pérot interferometers, all mounted on the same axis, permit a further separation of the bandpass obtained by the Ebert-Fastie. It may be shown that the resolving power remains at the level of 500 from $1200 \AA$ to $8000 \AA$.

The electronics must be quite sophisticated as different observational programmes must be foreseen and as spectra must be added channel per channel prior to telemetering. Three operating modes are considered 
- low resolution (100) monochromators scanning mode, interferometers off

- medium resolution (500) monochromators and interferometer alternate scanning mode

- surface mapping in four spectral ranges (all spectral devices stopped at a chosen position).

The total power required may be kept between the limits of 5 and $7 \mathrm{~W}$.

The total weight may drop as low as $4.2 \mathrm{~kg}$, if magnesium is used for the casing. If the total number of spectrographs is cut down to 3 or 2 , the total weight drops down to 3.2 and $2.4 \mathrm{~kg}$ respectively, but the interest of the concept is progressively decreasing.

\section{Reference}

Barth, C. A., Hord, C. W., Pearce, J. B., Kelly, K. K., Anderson, G. P., and Stewart, A. I.: 1971, J. Geophys. Res. 76, 2213. 